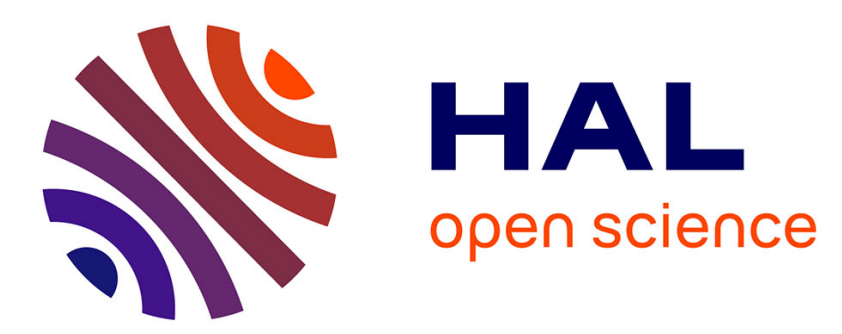

\title{
Profiling Children With Cerebral Visual Impairment Using Multiple Methods of Assessment to Aid in Differential Diagnosis
}

\author{
Amanda Lueck, Gordon Dutton, Sylvie Chokron
}

\section{To cite this version:}

Amanda Lueck, Gordon Dutton, Sylvie Chokron. Profiling Children With Cerebral Visual Impairment Using Multiple Methods of Assessment to Aid in Differential Diagnosis. Seminars in Pediatric Neurology, 2019, 31, pp.5-14. 10.1016/j.spen.2019.05.003 . hal-02401220

\author{
HAL Id: hal-02401220 \\ https://hal.science/hal-02401220
}

Submitted on 30 Nov 2020

HAL is a multi-disciplinary open access archive for the deposit and dissemination of scientific research documents, whether they are published or not. The documents may come from teaching and research institutions in France or abroad, or from public or private research centers.
L'archive ouverte pluridisciplinaire HAL, est destinée au dépôt et à la diffusion de documents scientifiques de niveau recherche, publiés ou non, émanant des établissements d'enseignement et de recherche français ou étrangers, des laboratoires publics ou privés. 


\title{
Profiling children with cerebral visual impairment using multiple methods of assessment
}

\author{
Amanda H. Lueck ${ }^{1^{*}}$, Gordon N. Dutton ${ }^{2}$ \& Sylvie Chokron ${ }^{3,4}$ \\ 1: Department of Special Education, San Francisco State University, San Francisco, CA, USA \\ 2: Department of Optometry and Visual Science, Glasgow Caledonian University, Cowcaddens \\ Rd, Glasgow. G4 0BA United Kingdom \\ 3: Unité Vision et Cognition, Fondation Opthalmologique Rothschild, Paris, France \\ 4: Laboratoire de Psychologie de la Perception, CNRS, UMR 8242 \& Université Paris \\ Descartes, Paris, France. \\ * Correspondence: \\ Amanda Lueck \\ amandal@sfsu.edu
}

Word Count: 7045

\begin{abstract}
Cerebral (cortical) visual impairment (CVI) is the primary cause of visual impairment in children in high-income countries. It is increasing globally due to improved life-saving measures for premature and full-term infants. Yet the consequences of this condition are only beginning to be understood and addressed. In contrast to visual impairment due to refractive error and disorders of the eye, there is limited public awareness of CVI, and the consequent impairment of a variety of visual capabilities may go unidentified, adversely affecting cognitive, motor, and social development. CVI refers to visual perception deficits not related to the peripheral (i.e., prechiasmatic) system disorder, but rather, to injury to brain areas serving assimilation, integration, and interpretation of visual information. According to the topography, site, and the extent of the pathology, the deficit may variably concern central visual functions, the visual field, perception of movement, visual analysis, visual exploration and attention, or visual memory, as well as visual guidance of movement. Each affected child has a unique clinical picture, which needs to be identified and individually profiled. This is probably the underlying reason that CVI is commonly underdiagnosed, especially in children, and, as a consequence, the full range of potential behavioral outcomes are not identified and adequately addressed. The present paper shows how the use of multiple methods of assessment can improve the understanding of children with CVI.
\end{abstract}

Keywords: Cerebral visual impairment; cortical visual impairment; learning disability; assessment; differential diagnosis, visual impairment 
Profiling children with cerebral visual impairment

\section{Cerebral Visual Impairments (CVI)}

\subsection{Definition, epidemiology, and etiology}

Cerebral visual impairment (also called cortical visual impairment) is a brain-based visual condition that affects pathways involved in processing incoming visual information via neural networks throughout the brain. In terms of epidemiology, CVI has become the main cause of childhood vision impairment in high-income countries and is on the rise in low-income countries (Dutton \& Lueck, 2015a, Kong et al., 2012). This huge increase in the proportion of visually impaired children having CVI in industrialized countries is due to improved measures to save at risk children born prematurely, and those who have prenatal or perinatal medical conditions, combined with more effective prevention and management of ocular causes of visual impairment. Indeed, cerebral anoxia (or hypoxia) and premature birth are the most common etiologies and associations with CVI (Fazzi et al, 2009; Jacobson \& Dutton, 2000; Pagliano et al., 2007, Shafer et al., 1986). In children with CVI, a history of preterm birth with or without cerebral anoxia or cerebral hypoxia is often reported. Although the term 'soft' neurological lesions, has been applied for affected children, the resulting neurological signs are far from 'soft' as they have functional implications that impair the child's ability to access information and learn (Chokron \& Dutton, 2016). In addition, traumatic brain injury, stroke, brain infection and genetic disorders represent the next most frequent causes of CVI (see Lueck \& Dutton, 2015a pp. xvii-xviii for review). The condition can be confounded with other disorders such as autism spectrum disorders, developmental coordination disorders, and learning disability (Chokron \& Dutton, 2016).

CVI describes deficiency in the functions of vision due to damage or malfunction of visual pathways and centers in the brain, specifically including the lateral geniculate bodies, the optic radiations, the occipital cortices and the visual associative areas, in any combination or degree. This may cause or be accentuated by associated disorders of eye movement control (Boot, et al., 2010; Dutton \& Lueck, 2015a; Fazzi et al., 2009, Ortibus et al., 2011). CVI is thus an umbrella term referring to visual and visual perceptual deficits that are not related to a peripheral (i.e. prechiasmatic) system abnormality, but rather to an injury of cerebral areas involved in the integration and interpretation of visual information, and guidance of movement through the environment. However, it has to be underscored that while CVI may be present in a child, the cerebral damage or dysfunction per se may not be detected using common brain imaging methods. Indeed, recent studies point to the fact that even in adults who suffer from CVI after stroke, for example, the lesion is not evident in more than $30 \%$ of the cases (Zhang et al., 2006a; 2006b). Thus, it is important to keep in mind that many children display the functional consequences of CVI, as we present below, even when the causative brain injury cannot be corroborated through conventional neuroimaging techniques.

Individuals with CVI may experience several kinds of difficulty when trying to move accurately through a visual space, or recognizing a simple visual object, and/or they may experience discomfort when faced with a complex visual scene. According to the topography and the size of the lesion, the deficit may concern the visual field, visual analysis, visual exploration, visual attention, visual memory and the accuracy of movement of the limbs and body.

Research on cerebral visual impairment has recently been emerging rapidly, advancing our understanding of the condition. CVI can be a stand-alone condition or can be accompanied by eye or optic nerve disorders (Fazzi et al., 2007; Jacobson \& Dutton, 2000). Children with CVI can range from those with normal to near normal ability to detect the detail of what they are viewing (or close

This is a provisional file, not the final typeset article 
Profiling children with cerebral visual impairment

to $20 / 20\{6 / 6\}$ vision), to those who have profoundly reduced visual clarity. Children's capabilities can span across learning levels to include those whose performance in school is equivalent to or even better than typical contemporaries, to those with profound learning challenges and additional disabilities (Lowery, et al., 2007).

As early damage to the brain is commonly diffuse and thus affects a range of functions, children with CVI often have concomitant neurological disorders (e.g., epilepsy, intellectual disability, cerebral palsy) that may increase the deleterious effects of CVI on cognitive, motor and social development. Indeed, several studies have been conducted in children with cerebral palsy to identify and characterize their associated CVI (Stiers et al., 2002; Fazzi et al., 2004). Overall these studies have shown that children with cerebral palsy have difficulties in visuo-perceptual, visuospatial, and visuo-constructive activities, irrespective of the level of visual acuity. It has thus been suggested that, in these children, the degree of the various deficits relates closely to the degree of reduction of white matter located below and within the corpus callosum, associated with impaired dorsal stream function, specifically in relation to spatial, motor, and attentional aspects of visual cognition (Fazzi et al., 2004).

\subsection{Semiology of CVI}

Before describing the semiology of children with CVI, it has to be emphasized that not only can CVI present with invisible deficits, but in addition, children who grow up with CVI may judge their vision to be "normal", and thus they do not systematically express a visual complaint, and so are asymptomatic for their disabling condition.

Consequently, their visual difficulties are mostly inferred from their behaviors and, among other deficits, can be expressed in terms of reduced learning abilities. However, as we will discuss further, there is a risk of confounding cerebral visual impairment and its consequences with other disorders that can affect behavioral, motor, and cognitive development. These may be the underlying reasons for under-diagnosis of CVI along with a concurrent massive increase, in industrialized countries, of undiagnosed children labeled as having behavioral as well as learning disabilities.

The timing, location, and extent of the causative pathology determine the severity of impairment in children with CVI (Jan \& Groenveld, 1990). As such, children can be broadly characterized as fitting into three groups (Dutton \& Lueck, 2015a): (1) children with profound visual impairment due to CVI, (2) children with CVI who have functionally useful vision and cognitive challenges, and (3) children with CVI who have functionally useful vision and who work at or near the expected academic level for their age group.

When a critical pathway, nucleus, or region in the brain is affected, many functions can be altered (Lezak, et al., 2012). Compromised vision can include issues associated with visual acuity impairment, visual field restriction, reduction in contrast sensitivity, impaired color vision, impaired visual fixation, oculomotor and exploratory strategy concerns, and focusing issues. Moreover, as the visual brain maps the surroundings, what appears to be clumsiness may be due to inaccuracy of movement due to less accurate 3D mapping of the surroundings, on account of CVI. Although often hidden, and for this reason under-diagnosed, disordered visual processing can affect children who have CVI in a variety of ways. Assessments must therefore address the behavioral manifestations of CVI, taking into account the additional full spectrum of potential typical behaviors that may arise in all children (Lueck \& Dutton, 2015a; Zihl \& Dutton, 2015). 
Profiling children with cerebral visual impairment

- There may be difficulty following fast movements such as rapid gestures (as in sign language) and transient facial expressions, rapid classroom demonstrations, rapid movement of children on skateboards or bicycles, or fast-moving animals. This difficulty may induce a fear of moving visual stimuli in children with CVI. Often when starting preschool, children may stay alone in the courtyard or in the playground, being diagnosed as presenting deficits in interacting with other children, whereas the cause of their loneliness is their difficulty processing moving stimuli or due to an effect described by parents and requiring further investigation designated as "looming" where children perceive that an object at a distance away is about to hit them (CVI Scotland $\left.2017^{1}\right)$.

- Difficulty working in crowded or cluttered environments that include a lot of visual or auditory distractions may be noted in some children. Not only can this be overwhelming but it can lead to CVI meltdowns where, as reported by parents and requiring further investigation, children become incapacitated when they cannot cope with the input overload from their environment (CVI Scotland, $2017^{1}$ ). Children who have this behavioral consequence of CVI may avoid busy environments or become anxious in them, choosing to stay on the sidelines when possible, or they may execute tasks poorly in busy situations they cannot avoid. To cope with such situations, some of them may close their eyes to suppress visual information.

- Despite apparently full visual fields, many children with CVI may not pay attention to objects in a particular portion of their field of vision (i.e., visual neglect), or may be unable to process several visual targets at the same time (due to visual extinction, or simultanagnosia). This type of deficit can induce severe difficulty in processing written language, which can be confounded with reading disabilities or dyslexia. It can also affect the ability to negotiate unfamiliar travel routes, to detect moving vehicles, people, or animals, or to visually search for a stimulus in a busy environment such as in a crowded drawer. A number of affected children discover that they can line up their toys so that they can find them again more readily.

- Children with CVI may have difficulty copying and drawing from memory. This can affect reading, map skills, and learning the principles of geometry.

- Some children may not be able to see faces and/or facial expressions, and thus may have difficulty recognizing people or 'reading' the emotions of others. This deficit is often confounded with autism or difficulty in interacting with people.

- There may be difficulty recognizing all or certain_objects, shapes, letters, or numbers. Often, children with CVI presenting with recognition disorders are mistakenly identified as children who do not pay attention or who present learning disabilities.

- Some children with CVI may get lost in space, and have difficulty remembering the locations of landmarks on their travel routes.

- Difficulty negotiating curbs and stairs can be found in some children.

- Accurate reach and grasp may be affected. Although in children with CVI these deficits in

${ }^{1}$ CVI Scotland. (2017.) Sharing and developing our understanding of CVI. http://cviscotland.org. Accessed 7 October 2017. 
Profiling children with cerebral visual impairment

visuo-motor coordination are linked to the visual deficit, there is often a confusion with developmental coordination disorder (See for review and discussion, Chokron \& Dutton, 2016.)

- Some children may have difficulty attending to auditory stimuli in addition to visual processing issues: some may be overwhelmed by competing and compelling stimuli in other sensory areas; others may not know where sounds are coming from, and some children may attend more readily to a particular cadence of speech or to speech that includes large changes in pitch that serve to attract auditory attention. (See Zihl \& Dutton, 2015.)

In all children, assessments must address issues associated with CVI specifically, but they must also address the full spectrum of potential behaviors that arise due to CVI including those that impact functional performance in various critical activities such as reading disorders, mathematical skills such as reading graphs, or following a prescribed travel route (Lueck \& Dutton, 2015a).

\section{Impact of CVI on cognitive and social development}

\subsection{CVI and learning disabilities}

Vision plays an essential role in the development of sensory-motor and cognitive skills (Mazeau, 2005; Chokron \& Dutton, 2016; Atkinson \& Braddick, 2007; Fraiberg, 1977; Fazzi, et al., 2010; Piaget, 1952, 1954, 1970). Vision has thus the capacity to coordinate all other perceptualsensory systems (Fraiberg, 1977). Visual experiences are first involved in the development of mental representations (in Fazzi et al., 2010; see Warren, 1984). Vision allows the child to learn though imitation, a process regarded as essential in human development (Carroll \& Bandura, 1982, 1987).

While studies on the impact of CVI upon development of young children are rare, there has been a good deal of research on the early development of children who are functionally blind due to ocular disabilities. Indeed, studies on blind children indicate a developmental delay, in comparison with typically sighted subjects, in various domains of motor development, especially self-initiated mobility, posture, and locomotion (Fraiberg, 1977; Tröster \& Brambring, 1992; Sonksen, 1993; Fazzi et al., 2002)._Fazzi et al. (2002) assessed early neuromotor development in children born with congenital blindness and showed that all their milestones were reached, albeit delayed. Similarly, children with multiple disabilities (i.e. cerebral palsy or intellectual disability) show a more marked postural delay (see also Fraiberg, 1997) as well as differences in the acquisition of object permanence (i.e. the ability to recognize that an object still exists even when it is no longer perceived), which is an indicator of cognitive development level (Bigelow, 1986; Fazzi et al., 2011).

In the same vein, studies on early visual deprivation (i.e., children born with central cataracts in both eyes and consequently deprived of visual input) indicate that early visual experience is necessary for the normal development of several visual functions, such as the ultimate development of normal visual acuity, contrast sensitivity for mid and high sensory frequencies, and some aspect of face perception (McKyton et al., 2015). Following these studies, and given the crucial role of vision in learning and cognitive development, it is evident that CVI, whatever the severity of visual impairment, will impact several areas of normal development and learning, especially if not identified and acted upon. Moreover, as CVI is the outcome of altered brain function, additional adverse effects of collateral impact can be foreseen.

\subsection{CVI and social interactions}


Profiling children with cerebral visual impairment

Visual impairment can affect the development of social responsiveness and communication. In the mother-child relationship during the first year of life, it is through sight that bonding and attachment are established. It has been suggested that if bonding is not, or is only partially established in infancy, this predisposes a child to future difficulties in interacting with people and the environment (Sonksen \& Dale 2002; Pawletko et al., 2015). It is essential to first determine whether spectacle correction for near results in engagement (Hyvarinen, 1995). However, when the visual deficit is diagnosed early enough in development, bonding can also be promoted by other non-visual sensory modalities including consistent, meaningful and contextualized verbalization and touch, to encourage attachment. This underscores the need for carefully matched and structured early intervention programs to support optimal development for these children.

There is indeed a close link between the visual system and the perception, experience and interpretation of emotion. It has been shown that the visual system contributes to amygdala activity which in turn modulates the responses of the ventral visual system involved in visual recognition (see Sabatinelli et al., 2009)]. Social abilities thus depend on several processes, including the ability to detect and interpret visual cues within interpersonal contexts (Dyck et al., 2004). It is also likely that delayed acquisition of communication skills can be a consequence of early visual impairment, rather than a primary disorder of socialization (Dale \& Sonksen, 2002; Fazzi et al., 2007; Fraiberg, 1977, Fazzi et al. 1999). Several studies have demonstrated that children with visual impairment are at risk for developing autistic-like behaviors (see for recent review Do et al., 2017).

CVI may thus interfere with the processing of visual information in all domains, and in addition, CVI may have mild to profound adverse effects on cognitive, motor and social development. Moreover, the potential consequences of other brain injuries must also be kept in mind. It is thus important to understand and document, through careful assessment, the full impact of CVI in children who have this condition to optimize their participation and learning within educational settings.

\section{Early identification of children with cerebral visual impairment}

Identification of children with cerebral visual impairment is vital for the provision of appropriate training for parents and services that optimize development and skill acquisition, as well as participation in school, home, and community life to the fullest extent possible. The need for early diagnosis and characterization of visual impairments, including cerebral visual impairments, in young children is well-recognized (e.g., Struble et al., 2016). This can lead to targeted interventions at younger ages, and improved outcomes in later years (Chokron \& Dutton, 2016).

Unfortunately, a number of the behavioral manifestations of CVI can be missed or misinterpreted if not understood within the context of damage to the visual brain (Dutton \& Lueck, 2015a). An inability to complete pre-academic or academic tasks, for example, may have been fully attributed to other learning or behavioral issues (Pawletko et al., 2015) when, in fact, it may be fully or partially related to CVI. In addition, cerebral visual impairment can be difficult to uncover when visual processing concerns are the primary manifestation of the condition (Lowery et al., 2007), and when they are coupled with normal or near normal visual acuity. Furthermore, visual processing concerns associated with CVI may not be discovered until a child attends primary school when more subtle features, particularly those related to reading, are noted (Cavézian et al., 2010). In addition, the range of effects of CVI may be overlooked in children who have additional disabilities (e.g., Ego, et al, 2015) or in children who have profound vision impairment, (Dutton \& Lueck, 2015b). In such instances, behavioral manifestations associated with impaired visual functions (e.g., visual acuity,

This is a provisional file, not the final typeset article 
Profiling children with cerebral visual impairment

visual field, contrast sensitivity deficits), and more obvious areas of visual processing (e.g., inability to look and reach at the same time; the need for reduced visual clutter) may be assumed to be the only ones in operation, overshadowing additional, but more subtle expressions of the condition that can adversely affect visual and overall performance as children mature and are required to respond to greater visual demands for learning.

\section{Goals of assessment of children with CVI in educational settings}

Assessment is a key component of educational programs, and in educational settings, assessment can have multiple goals. These range from initial screening that will result in follow-up evaluations to aid in early identification, in-depth examination related to identification and diagnosis, and gathering of functional information about performance in order to make optimal decisions about students (Salvia et al., 2013; Ysseldyke et al., 2009). The types of assessment conducted must take into account the ways in which assessment outcomes are to be applied for identification, intervention, and decision-making purposes.

\section{Use of multiple methods of assessment}

CVI is a complex condition, and assessment methods must ensure that both readily apparent and subtle manifestations of CVI are uncovered and documented. To accomplish this, there is a need to investigate potential outcomes of CVI within a variety of activities and tasks that provide data from different perspectives using multiple methods. This can be considered a variant of the research approach called "Triangulation of Data", where multiple data sources are used to produce understanding that captures different aspects of the same phenomenon. Even when there may be inconsistencies across data sources, such inconsistencies are a source of strength as they provide an avenue to explore deeper meaning in the data (Nokleby, 2011; Patton, 2002). For children with a complex condition such as CVI, using multiple methods of investigation is the necessary course of action because, at this time, one system that evaluates all possible factors associated with CVI does not exist.

In addition to assessment of developmental progress and skills in various activities in the school, home, and community, an important area to assess for children with CVI and their families, centers around knowing about, understanding and accepting the condition (Morse \& Morse, 2015). Understanding and acceptance can promote positive circumstances that lead to empowerment, selfefficacy, and self-determination.

A comprehensive assessment includes contributions from a multidisciplinary team including the family, and when possible, the child. The make-up of a multidisciplinary team will vary for each child, and can include both educational and medical practitioners, with membership varying in different regions.

Given the various goals of assessment and the range of the effects of CVI, an assessment process that includes a variety of diagnostic techniques can prove highly useful (Walthes et al., 2015). In clinical practice, this is best founded upon detailed prior knowledge of the subject to correctly interpret the multiple methods of assessment that can include: (1) interview/expert historytaking and interpretation, (2) observation of behavior, (3) formal/informal assessment of visual functions and functional vision, (4) evaluation of skills across developmental and educational domains in school, home, community, (5) analysis of the physical environment and instructional material, and (6) diagnostic teaching (i. e., where intervention methods and results are carefully 
Profiling children with cerebral visual impairment

332

333

334

335

336

337

338

339

340

341

342

343

344

345

346

347

348

349

350

351

352

353

354

355

356

357

358

359

360

361

362

363

364

365

366

367

368

369

370

371

372

373

374

375

376

monitored and recorded, with results used to modify or maintain ongoing interventions (Lueck \& Dutton, 2015b; Koenig \& Holbrook, 1989)). The latter will be discussed in conjunction with intervention later in this paper.

\subsection{Interview/history-taking}

Interviews and history-taking are critical to the evaluation of children with CVI because the condition often goes undiagnosed, and children are frequently given alternative diagnoses that center around behaviors attributed to cognitive, motor, or social developmental issues. These issues may actually be outcomes of CVI, however. When this occurs, there is a major risk of diagnostic confusion between CVI and learning disabilities and/or motor disabilities and/or social interaction deficits such as autistic traits. In this way, medical history-taking, examination and (when indicated) brain imaging, need to be sufficiently thorough and comprehensive to uncover the nature, etiology, probable location and origin of the cerebral pathology, while habilitational history-taking focuses upon the nature and origins of the limitations caused by the visual impairment, the behavioral outcomes, and their impact.

Moreover, in both education and medical settings, interviews and history-taking can also be ways to determine a broad spectrum of adverse effects of CVI (Zihl \& Dutton, 2015; Dutton, (2017); Dutton \& Lueck, 2015a; Ortibus et al., 2011). Use of non-leading, open-ended targeted questions can reveal many behavioral manifestations of the condition (Dutton, 2015; Zihl \& Dutton, 2015).

Short versions of history-taking questionnaires used in careful combination with medical history and other medical information have been found to help suspect and support a diagnosis of CVI, particularly for children with normal/near-normal visual acuity (van Genderen et al, 2012). In addition, a more expanded history taking method that has been developed, not to diagnose CVI, but to identify patterns of expression of the condition that can be tied to intervention options is being made available (Dutton, 2017²; Macintyre-Beon et al., 2012; Mitry et al., 2016).

\subsection{Observation}

The ways in which children with CVI approach and complete various tasks (observing motor, cognitive, and communicative behaviors within them); perform with different materials and in different environments; and respond in social situations and to social cues provide abundant insights into the ways in which a child's CVI manifests. Interpretation of observed behaviors requires an understanding of development and learning as well as the ways in which CVI can affect function. Observations can be completed independently from the administration of formal or informal assessments, or they can be an integral part of these methods. For example, the observation that a child uses his left hand, rather than his right hand, to sort clothes on his left side and uses his right hand to sort clothes on his right side can provide important information about how the child integrates vision and motor skills. It may also be noted that the child spreads the clothes out to sort them rather than pulling them out from a pile of clothes. These observational findings provide valuable insights into how the child operates and learns. Determining factors that motivate children with CVI to learn via observation (as well as interview) can help to establish appropriate intervention strategies that help maintain the child's interest in learning tasks, and thereby maximize positive

2 Dutton, G.N. Insight- approaches for visual perceptual difficulties. Web document in preparation.

This is a provisional file, not the final typeset article 
Profiling children with cerebral visual impairment

377

378

379

380

381

382

383

384

385

386

387

388

389

390

391

392

393

394

395

396

397

398

399

400

401

402

403

404

405

406

407

408

409

410

411

412

413

414

415

416

417

418

419

420

421

422

423

424

425

learning outcomes. Various observational protocols are available for recording observations of children with visual impairments or, more specifically, CVI (e.g., Lueck \& Dutton, 2015b; RomanLantzy, 2010; Steendam, 2015). But a keen observer will be alert to behaviors and conditions that are not listed on available protocols as well, since CVI can manifest in so many ways that are unique to individual children. These attendant behaviors can surface during more formal testing situations, and when they do, they can provide helpful clues toward understanding the nature of a child's CVI.

\subsection{Assessment of visual functions and functional vision in children with CVI}

Perhaps the most researched and applied areas of assessment for children with visual impairment in general are those related to the evaluation of visual functions and functional vision. Fortunately, a small number of highly specific, standardized assessment tools associated with early diagnosis of visual perception disorders in young children are available, and more protocols are being developed. Many of these tools or methods target early diagnosis/identification (e.g., Atkinson et al., 2002; Cavézian et al., 2010; Garcia-Ormaechea et al., 2014; Ortibus et al., 2009; Ortibus et al., 2016; Pel et al., 2016; Vancleef et al., 2009), while others are connected to curricular applications and compensatory adaptations (e.g., Dutton, 2014 Macintyre-Beon et al., 2012; Mitry et al., 2016; Roman-Lantzy, 2007).

Methods to evaluate the visual capabilities of young children with visual impairments associated with their visual functions such as visual acuity, visual field, contrast sensitivity, oculomotor mechanisms, and accommodation have been developed and refined over the past 30 years (Lueck, 2004; Corn \& Erin, 2010; Duckman, 2006;). These behavioral and electro-diagnostic techniques have been further refined for application to children who have CVI (Kran \& Mayer, 2015; Hyvarinen \& Jacob, 2011; Dutton \& Bax, 2010; Watson et al., 2010). Furthermore, diagnostic methods associated with the evaluation of visual processing concerns for children who specifically have CVI have received attention (Hyvarinen \& Jacob, 2011; Zuidhoek et al., 2015). Application of neuropsychological assessment methods for children with CVI requires careful attention, since norms on current standardized tests have yet to include or target children with CVI within their population samples (Zuidhoek, 2015; et al., 2015).

\subsection{Evaluation of skills across developmental and educational domains}

While it is imperative to pinpoint and isolate specific consequences of CVI for individual children, the potential effects of CVI cannot be considered in isolation from overall learning, daily living and social activities in the school, home, and community (Lueck \& Dutton, 2015b). As a result, systematic, multidisciplinary and multidimensional assessment approaches are needed that:

- Determine the consequences of both ocular and brain-based visual impairment (Kran \& Mayer, 2015),

- Recognize the role of executive functions, the issues related to attention and their role in behavioral outcomes for children with CVI (Zuidhoek, 2015; Zuidhoek et al., 2015; Zihl \& Dutton, 2015),

- Pinpoint areas of strength as well as challenges (Hyvarinen, 2003),

- Investigate the possibility of attendant disorders (Pawletko et al., 2015),

- Look at vision within the context of the whole child and the activities they perform in various settings (Creamer O’Brien \& Martyn, 2015; Lueck et al., 1999; Lueck \& Dutton, 2015b).

\section{5 Analysis of the physical environment, instructional materials, and children's interests}


Profiling children with cerebral visual impairment

\section{and motivators}

CVI requires interventions that address activities in school, home, and community environments, as well as appropriate formats for methods and materials that promote development and learning. The performance of children with CVI can vary greatly depending upon conditions in the physical environment, biobehavioral state, types of materials used in the instructional program, performance requirements of targeted intervention tasks, and familiarity with materials/locales/evaluators, and task motivation (Lueck \& Dutton, 2015b). These broader external and internal elements associated with the learning environment and children's physical and mental states may influence performance outcomes and need to be documented throughout the assessment and intervention process to identify factors that will lead to optimal outcomes. Materials designed to systematically characterize these important elements are emerging (e.g., Roman, 2010; Tietjen, 2015; Sheline, $n . d^{3}$.). Furthermore, children's interests and motivators must be taken into account in the development of instructional goals and strategies. Tasks must be accessible, age appropriate, meaningful and enjoyable in order to engage and encourage children to participate and learn.

\section{Towards a process that ties assessment to intervention}

Currently, a single instrument that pinpoints and characterizes all possible behavioral outcomes of CVI is not available due to the scope of expression of the disorder as well as the unique ways in which CVI can arise in individual children. Until more refined and overarching methods of assessment are available (and perhaps this will never be possible with such a complex set of conditions), a comprehensive understanding of the effects of CVI in individual children requires a synthesis of the methods discussed earlier.

The coordination of such methods, based upon a firm knowledge of potential effects of CVI, coupled with a detailed medical and educational history, along with an understanding of children's and family's capabilities, desires and interests, can assist in the interpretation of performance outcomes. This approach is applied to evaluate the ways in which a child responds to a variety of tasks in a variety of environments with a variety of materials. This informed "observation of process", rather than reliance on final outcome scores, helps construct a detailed and comprehensive picture of a child's functional capabilities that can guide ongoing intervention approaches (Zuidhoek et al., 2015). The observation of process can be evaluated within some available tools or methods developed specifically for children with CVI but also within other types of assessment or developmental protocols designed for children who have visual impairments (e.g., Anderson et al., 2007; Lueck et al., 2008; Roman-Lantzy, 2007).

Future work on assessment paradigms that focus on process and encompass a broad range of effects of CVI will hopefully be developed and refined as knowledge of CVI expands. Development of reliable and valid assessment tools takes time. Until such tools are available, practitioners must carefully evaluate a child's performance using items within existing formal and informal assessment and intervention protocols in the context of their knowledge of CVI (e.g., Zuidhoek et al., 2015). Results can point the way toward task modifications that can then be incorporated into instructional programs to enhance a child's engagement in task activities and promote learning. Instruments related to overall development for young children as well as tools designed to measure pre-academic

\footnotetext{
3 Sheline, D. Strategies for Improving Literacy Skills in Students with CVI. Perkins E-Learning http://www.perkinselearning.org/videos/webinar/strategies-for-improving-literacy-skills-in-students-cvi. Accessed 13 October, 2016.
} 
Profiling children with cerebral visual impairment

471

472

473

474

475

476

477

478

479

480

481

482

483

484

485

486

487

488

489

490

491

492

493

494

495

496

497

498

499

500

501

502

503

504

505

506

507

508

509

510

511

512

513

514

515

516

517

518

519

skills, reading, mathematics and other curricular areas can be administered and scrutinized in this way. Emphasis is therefore placed on observation of how tasks are approached and completed, looking for behavioral variants that may be consistent across tasks and associated with CVI, given the particular child's medical history.

When formal assessment items require any form of adaptation or modification, and children with visual impairments have not been included in the standardization protocols, it is generally accepted that standardized scores cannot be applied without additional validation efforts that includes these children (Morash \& McKerracher, 2017). It has, however, also been proposed that the need for additional standardization studies for this population may depend upon the types of adaptations introduced since some may not significantly alter the degree of difficulty of test items (Ruiter et al., 2011). For children who have CVI, it must be determined whether or not particular adaptations or modifications alter the underlying skills and behaviors tapped by the original item. For instance, inability to perceive more than one or two items at once, due to simultanagnosia, confounds any attempt to assess the ability to find and match paired items amongst other similar items as well as performing any test that comprises several visual stimuli to process.

\subsection{Hypothetical case study as an example of evaluation of process}

To clarify the evaluation of process rather than outcome, a task item has been broken down to evaluate process for a 7-year-old child with CVI who reads at grade level. This hypothetical child has been diagnosed with hypoxic-ischemia encephalopathy at full-term birth (a brain injury due to reduced blood flow and oxygen to the brain) and has difficulty copying figures. Rather than making a summary statement that the child cannot copy outlined figures of shapes and capital letters, an investigator has decided to explore how the child approaches copying tasks by adjusting the copying activities to clarify precisely where the copying issues arise.

Many factors could be involved when a child is asked to copy a figure such as: (1) understanding the instructions, (2) detecting and paying attention to the whole visual field, (3) being able to fixate on and encompass the figure to be copied, (4) being able to process the space on the sheet of paper and the figure, (5) size of the figures and the working distance of the figure, (6) level of illumination, (7) color of the lines and/or the color of the background, (8) contrast levels of the figure and background, (9) complexity of the figure or array of figures (e.g., a diagonal line versus a complete triangle versus an octagon; one figure on a line versus an array of figures), (10) presence or absence of visual distractors and the nature of those distractors, (11) auditory and other sensory distractors in the physical environment, (12) child's ability to process the global shape as well as the local details, (13) child's motor control in copying lines in different directions and connecting them without overlap, (14) use of line markers or other methods to draw a child's attention to the figure, (15) effect of modeling by the examiner who might draw the full figure immediately prior to copying or who might draw successive parts of a figure for the child to copy, (16) child's familiarity with the figure in target tasks, (17) spatial placement of figures that might indicate any visual field loss or visual neglect (such as finding figures on the left right or bottom of the page visually following along lines of figures or locating the beginning of a line of figures), (18) ability of the child to scan from right to left, left to right, or from top to bottom, bottom to top of an array of figures and to use an adequate and strategic visual exploration method, (19) ability of the child to shift gaze from the model figure to the paper to create a copy, (20) ability of the child to hold the pen and visually control his/her drawing, and (21) ability of the child to find the target of interest each time, as gaze shifts back and forth. 
Profiling children with cerebral visual impairment

520

521

522

523

524

525

526

527

528

529

530

531

532

533

534

535

536

537

538

539

540

541

542

543

544

545

546

547

548

549

550

551

552

553

554

555

556

557

558

559

560

561

562

563

564

565

566

567

Deconstructing items on protocols or in activities or tasks in this manner and adjusting task components can be extremely helpful in understanding, more precisely, how a child with CVI operates and lead to important intervention recommendations. It will also lead to a clearer understanding by the child and family about how the child sees best and learns. Analyzing a child's errors and response modes may, in turn, lead to the introduction of alternative, easier methods that can then be evaluated to determine how effective they are. For example, in most children with CVI, performing a task with no visual control (eyes closed) greatly improves the performance in motor tasks even when some precision is required such as in writing tasks (Chokron \& Dutton, 2016).

\section{Diagnostic teaching}

Within an instructional program, learning techniques and instructional adaptations can be monitored for impact on performance. Approaches can be maintained or revised based on observations within targeted tasks as part of a diagnostic teaching paradigm (Koenig \& Holbrook, 1989; Lueck \& Dutton, 2015b; Lueck \& Dutton, 2015c). Hypotheses about children are implemented using teaching methods derived from assessments. Performance outcomes are noted, and revisions to instruction are made based upon these outcomes. As intervention programs are implemented, careful monitoring of materials, methods, and environment are continued, with further refinement of intervention techniques as new knowledge of the child emerges and as the child's performance and learning environment change over time.

\section{Issues in assessment for children with cerebral visual impairment}

The complexity of CVI, a brain-based disorder, raises a number of issues that must be addressed throughout a child's school career:

- Systematic methods are needed to identify affected children in schools, including those with normal to near-normal visual acuity. This includes close monitoring and assessment of children born prematurely and those with very low birthweight who may be at risk of impairments in visual perceptive abilities, particularly in visual-spatial perception, as well as visual-motor integration deficits (Geldof et al., 2012).

- Children with CVI must be differentiated from those who have Autism Spectrum Disorder, Attention-Deficit/Hyperactivity Disorder (ADHD), Learning Disability, or Developmental Coordination Disorder (DCD), or children who may have one of these conditions in addition to CVI (Pawletko et al., 2015; Chokron \& Dutton, 2016).

- Children who have profound visual impairments are most likely to be identified at early ages due to the severity and more visible nature of their conditions. However, more subtle effects of their disorders may not be recognized as these children move through the education system, since they can be masked by other issues. Thus, appropriate educational interventions to address these more "hidden" impairments may not be addressed (Lueck \& Dutton, 2015b; Morse \& Morse, 2015).

- In addition, the presence of CVI can be masked in children who have visual disorders such as amblyopia or strabismus, because these diagnoses are the focus of attention.

- Investigation of subtle sequelae of the disorder must be instituted for children who have

This is a provisional file, not the final typeset article 
Profiling children with cerebral visual impairment

multiple disabilities including CVI. For example, Ego et al., (2015), reviewed visual-perceptual skills for children with cerebral palsy (CP) and recommended that the impairment of visualperceptual skills be considered a core disorder within the CP syndrome, requiring the adoption of a more systematic approach to the evaluation of these skills within neuropsychological testing of the CP population.

- Understanding the visual capabilities of young children with CVI, including the array of visual perceptual skills that can be affected is critical. How these compromised skills might affect overall development requires additional research along with insightful observation and assessment when using existing resources (Fazzi et al., 2015).

- The impact of CVI throughout a child's entire school program and environments, as well as in home and community needs to be fully addressed (Lueck \& Dutton, 2015b), otherwise these children might be failing where they could otherwise succeed given appropriate understanding of their condition and intervention methods.

- Practitioners require training in understanding the breadth and depth of effects of CVI so that they can understand how children function on assessment and intervention tasks, make appropriate task adaptations and goals, and thereby maximize learning without placing undue stress or demands on children with this condition.

- Parents and caregivers must be taught in detail about how their children see, and how to optimize their parenting, as well as working closely with schools to ensure that the child's school and home experiences are coordinated.

\section{Discussion}

Throughout this paper, we have presented ways in which vision has a cardinal role in a child's visual development and the extent to which CVI can compromise learning, cognitive and motor development, and interaction with the outside world. The importance of the visual modality throughout all skills learned in early life is illustrated.

Consequently, given the role played by vision in childhood development, a number of foundational skill sets can be disrupted when vision cannot make its typical contributions to perception, visual-motor coordination or relationships toward the outside world. Along these lines, it has been recommended that a neuropsychological approach should be adopted that systematically evaluates, in detail, the cognitive, motor, and alternative sensory processes involved in task performance (Pennington, 2008). Whenever a complex neuropsychological task is given to a child, one should always first ensure that all elements of the task are - or have been rendered - visible, as well as question the nature of the cognitive and perceptual processes entailed, so as to decipher the nature of the child's problem solving and performance capabilities (Chokron \& Dutton, 2016; Zuidhoek et al., 2015). When encountering a child with a deficit in cognitive, motor, or social development, a range of visual capabilities (not just visual acuity) must first be evaluated. This is essential to avoid confusion between CVI, with its impact upon cognitive, motor, and social development, (including the ability to learn and acquire Theory of Mind). This will help ensure that the diagnosis of CVI is not erroneously confounded with autistic traits or intellectual deficiency (Pawletko et al., 2015; Chokron \& Dutton, 2016). 
CVI can impair the capacity to perform a large variety of tasks, giving the illusion of multiple deficits when, in fact the entire cause is the visual impairment. This is the reason why, the deleterious effects of CVI on social interaction, reading, writing, calculating, visuo-motor coordination, and so on, can easily be confounded with such conditions as autistic spectrum disorder, dyslexia, dysgraphia, dyscalculia and developmental coordination disorder. The complex variety of behavioral outcomes of the condition can best be discovered through the use of multiple methods of assessment at this time.

In conclusion, $\mathrm{CVI}$ is not a single diagnosis, it is an umbrella term for all types of visual impairment due to brain injury or dysfunction. Each affected child has his or her own unique clinical picture, which needs to be identified and individually profiled. However, in contrast to visual impairment due to refractive error and disorders of the eye, there is limited public awareness of CVI, and the consequent impairment of a variety of visual capabilities may go unidentified (Chokron \& Dutton, 2016). Differentiation between CVI and other neurodevelopmental disabilities is thus crucial, and methods for achieving this aim have yet to be systematized.

644

On the other hand, while a 'learning disability' can be the 'visible' deficit in a child, potential underlying CVI may be present and invisible, often going unnoticed even by the child, who grows up unaware that his or her vision is atypical since it is what the child has always known (Chokron \& Dutton, 2016). There is thus an urgent need for greater understanding of these impairments to enable better and earlier diagnosis and intervention. Optimal characterization of the interplay between CVI and the various developmental disorders, especially developmental coordination disorder and autism spectrum disorders, which share a similar semiology and etiology, is essential (Chokron \& Dutton, 2016). Greater knowledge and awareness of CVI in all its presentations is likely to significantly improve clinical practice and reshape fundamental theories concerning visual and cognitive development in typically and atypically developing children. 
Profiling children with cerebral visual impairment

645

646

647

648

649

650

651

652

653

654

655

656

657

658

659

660

661

662

663

664

665

666

667

668

669

670

671

672

673

674

675

676

677

678

679

680

681

682

683

684

685

686

687

688

689

690

691

\section{References}

Anderson, S., Boigon, S., Davis, K., deWaard, C. (2007). The Oregon project for preschool children who are blind or visually impaired. Sixth edition. Medford, OR: Southern Oregon Educational Service District.

Atkinson, J., Anker, S., Rae, C., Hughes, C., \& Braddick, O., (2002). A test battery of child development for examining functional vision (ABCDEFV). Strabismus, 10 (4), 245-69.

https://doi.org/10.1076/stra.10.4.245.13831

Atkinson \& Braddick, (2007). Visual and visuocognitive development in children born very prematurely. Progress in Brain Research, 164, 123-149. https://doi.org/10.1016/S0079$\underline{6123(07) 64007-2}$

Bigelow, A.E. (1986). The development of reaching in blind children. British Journal of Developmental Psychology, 4, 355-366. https://doi.org/10.1111/j.2044-835X.1986.tb01031.x

Boot, F. H., Pel, J. J., Van der Steen, J., Evenhuis, H. M. (2010). Cerebral visual Impairment: Which perceptive visual dysfunctions can be expected in children with brain damage? A systematic review. Research in Developmental Disabilities, 31, 1149-1159.

https://doi.org/10.1016/j.ridd.2010.08.001

Carroll, W.R., \& Bandura, A._(1982). The role of visual monitoring in observational learning of action patterns: making the unobservable observable. Journal of Motor Behavior, 14, 153-67. https://doi.org/10.1080/00222895.1982.10735270

Carroll, W.R., \& Bandura, A._(1987). Translating cognition into action: the role of visual guidance in observational learning. Journal of Motor Behavior. 19, 385-98.

https://doi.org/10.1080/00222895.1987.10735419

Cavézian C., Vilayphonh M., De Agostini, M., Vasseur, V., Watier, L., Kazandjian S., Laloum, \& L., Chokron, S. (2010), Assessment of visuo-attentional abilities in young children with or without visual disorder: Toward a systematic screening in the general population. Research In Developmental Disabilities, 31, 1102-1108. https://doi.org/10.1016/j.ridd.2010.03.006

Chokron S., \& Dutton, G.N. (2016). Impact of cerebral visual impairments on motor skills: Implications for developmental coordination disorders. Frontiers in Psychology, 7, 1-15. https://doi.org/10.3389/fpsyg.2016.01471

Corn \& Erin, (Eds.) (2010). Foundations of low vision: Clinical and functional perspectives. Second Edition. New York, NY: AFB Press.

Creamer O’Brien, \& Martyn, A. (2015). Assessment linked to interventions: Independent living skills. In A.H. Lueck \& G.N. Dutton (Eds.), Vision and the brain: Understanding cerebral visual impairment in children (pp. 435-449). New York, NY: AFB Press. 
Profiling children with cerebral visual impairment

692

693

694

695

696

697

698

699

700

701

702

703

704

705

706

707

708

709

710

711

712

713

714

715

716

717

718

719

720

721

722

723

724

725

726

727

728

729

730

731

732

733

734

735

736

737

738

739

740
Dale, N., \& Sonksen, P. (2002). Developmental outcome, including setback, in young children with severe visual impairment. Developmental Medicine \& Child Neurology, 44, 613-622. https://doi.org/ 10.1111/j.1469-8749.2002.tb00846.x

Do, B., Lynch, P., Macris, E.M., Smith, B., Stavrinakis, S., Quinn, S., \& Constable, P.A. (2017). Systematic review and meta-analysis of the association of autism spectrum disorder in visually or hearing impaired children. Ophthalmic Physiological Optics, 37, 212-224.

https://doi.org/10.3389/fpsyg.2016.01471

Duckman, R.H. (Ed.). (2006). Visual development, diagnosis, \& treatment of the pediatric patient. Philadelphia, PA: Dutton, Lippincott, Williams, \& Wilkins.

Dutton, G.N. (2015). Assessment of functional vision History taking in children with CVI. In A.H. Lueck \& G.N. Dutton (Eds.), Vision and the brain: Understanding cerebral visual impairment in children. (pp 261-276). New York, NY: AFB Press.

Dutton, G.N., \& Bax, M. (2010). Visual impairment in children due to damage to the brain. Clinics in Developmental Medicine No. 186. London: MacKeith Press.

Dutton G.N., \& Lueck, A.H. (2015a). Impairment of vision due to damage to the brain. In A.H. Lueck \& G.N. Dutton (Eds.), Vision and the brain: Understanding cerebral visual impairment in children. (pp 3-20). New York, NY: AFB Press.

Dutton G.N., \& Lueck, A.H. (2015b). Cerebral visual impairment and cerebral blindness in very young children: Connecting assessment to intervention. In A.H. Lueck \& G.N. Dutton(Eds.), Vision and the brain: Understanding cerebral visual impairment in children (pp. 537- 571). New York, NY: AFB Press.

Dyck, M.J., Farrugia, C., Shochet, I.M., \& Holmes-Brown, M. (2004. Emotion recognition/understanding ability in hearing or vision-impaired children: do sounds, sights, or words make the difference? Journal of Child Psychology \& Psychiatry, 45, 789-800.

https://doi.org/10.1111/j.1469-7610.2004.00272.x

Ego, A., Lidzba, K., Brovedani, P., Belmonti, V., Gonzalez-Monge, S., Boudia, B., Ritz, A., \& Cans, C. (2015). Visual-perceptual impairment in children with cerebral palsy: A systematic review. Developmental Medicine \& Child Neurology, 57, 48-51. https://doi.org/10.1111/dmcn.12687

Fazzi, E., Lanners. J., Danov, S., Ferrarri-Ginevra, O., Gheza, C., Luparia, A., Balottin, U., Lanzi, G. (1999). Stereotyped behaviours in blind children. Brain \& Development, 21, 522-8. https://doi.org/10.1016/S0387-7604(99)00059-5

Fazzi E., Lanners J., Ferrari-Ginervra O, Achille C., Luparia, A., Signorini S et al, (2002). Gross motor development and reach on sound as critical tools for the development of the blind child. Brain \& Development, 24, 269-275. https://doi.org/10.1016/S0387-7604(02)00021-9

Fazzi, E, Bova SM, Uggetti C, Signorini SG, Bianchi PE, Maraucci I, Zoppello M, Lanzi G. (2004). Visual-perceptual impairment in children with periventricular leukomalacia. Brain \& Development, 26, 506-12. https://doi.org/10.1016/j.braindev.2004.02.002 
Profiling children with cerebral visual impairment

741

742

743

744

745

746

747

748

749

750

751

752

753

754

755

756

757

758

759

760

761

762

763

764

765

766

767

768

769

770

771

772

773

774

775

776

777

778

779

780

781

782

783

784

785

786

787

788

789
Fazzi, E., Bova, S., Giovenzana, A., Signorini, S., Uggetti, C., \& Bianchi, P. (2009). Cognitive visual dysfunctions in preterm children with periventricular leukomalacia. Developmental Medicine \& Child Neurology, 51, 974-981. https://doi.org/10.1111/j.1469-8749.2009.03272.x

Fazzi E., Signorini S.G., \& Lanners J. (2010). The effect of impaired vision on development. In G. Dutton \& M. Bax (Eds.), Visual impairment in children due to damage to the brain (pp. 162-173). London: McKeith Press.

Fazzi, E., Signorini, S.G., Bova, S.M., La Piana, R., Ondei, P., Bertone, C., Misefari, W., \& Bianchi, P.E. (2007). Spectrum of visual disorders in children with cerebral visual impairment. Journal of Child Neurology, 22, 294-301. https://doi.org/10.1177/08830738070220030801

Fazzi, E., Signorini, S.G., Bomba, M., Luparia, A., \& Lanners, J., \& Balottin, U. (2011). Reach on sound: A key to object permanence in visually impaired children. Early Human Development, 87, 289-296. https://doi.org/10.1016/j.earlhumdev.2011.01.032

Fazzi, E., Molinaro, A., \& Hartmann, E. (2015). The potential impact of visual impairment and CVI on development. In A.H. Lueck \& G.N. Dutton (Eds.), Vision and the brain: Understanding cerebral visual impairment in children (pp. 83-105). New York, NY: AFB Press.

Fraiberg, S. (Ed.). (1977). Insights from the blind: Comparative studies of blind and sighted infants. New York, NY: Basic Books.

Garcia-Ormaechea, I., Gonzalez, I., Dupla, M., Andres, W., Pueyo, V. (2014). Validation of the Preverbal Visual Assessment (PreViAs) Questionnaire. Early Human Development, 90, 635- 638. https://doi.org/10.1016/j.earlhumdev.2014.08.002

Geldof, C.J.A., van Wassernaer, A.G., de Kietviet, J.E., Kok, J.H., \& Oosertaan, J. (2012). Visual perception and visual-motor integration in very preterm and/or very low birth weight children: A meta-analysis. Research in Developmental Disabilities, 33, 726-736.

https://doi.org/10.1016/j.ridd.2011.08.025

Hyvarinen, L. (1995). Considerations in the evaluation and treatment of the child with low vision. American Journal of Occupational Therapy, 49, 891-7. https://doi:10.5014/ajot.49.9.891

Hyvarinen, L. (2003). Assessment of CVI: L. Hyvarinen's lectures at San Francisco State. 15 November, 2003. http://www.lea-test.fi/en/assessme/sfracvilect/index.html. Accessed 7 October 2017.

Hyvarinen, L., \& Jacob, N. (2011). What and how does this child see. Helsinki, Finland: VISTEST Ltd.

Jacobson, L. K., \& Dutton, G.N. (2000). Periventricular leukomalacia: An important cause of visual and ocular motility dysfunction in children. Survey of Ophthalmology. 45, 1-13. https://doi.org/10.1016/S0039-6257(00)00134-X

Jan, J.E., \& Groenveld, M. (1990). Visual behaviors and adaptations associated with cortical and ocular impairment in children. Journal of Visual Impairment \& Blindness, 87, 101-105. 
Profiling children with cerebral visual impairment

790

791

792

793

794

795

796

797

798

799

800

801

802

803

804

805

806

807

808

809

810

811

812

813

814

815

816

817

818

819

820

821

822

823

824

825

826

827

828

829

830

831

832

833

834

835

836

Koenig, A J. \& Holbrook, M. C (1989). Determining reading medium for students with visual impairments: A diagnostic teaching approach. Journal of Visual Impairment \& Blindness, 83, 296302.

Kong, L., Fry, M., Al-Samarraie, M. (2012). An update on progress and the changing epidemiology of causes of childhood blindness worldwide. Journal of the American Academy of Pediatric Ophthalmology \& Strabismus, 16, 501-507. https://doi.org/10.1016/j.jaapos.2012.09.004

Kran, B.S., \& Mayer, D. L. (2015). Assessment of visual function and functional vision: Clinical assessment and suggested methods. In A.H. Lueck \& G.N. Dutton (Eds.), Vision and the brain: Understanding cerebral visual impairment in children (pp.277-342). New York, NY: AFB Press.

Lezak, M.D., Howieson, D.B., Bigler, E.D., Tranel, D. (2012). Neuropsychological Assessment, 5th edition. New York, NY: Oxford University Press.

Lowery, R.S., Atkinson, D., \& Lambert, S.R. (2007). Cryptic cerebral visual impairment in children. British Journal of Ophthalmology, 90, 960-963. https://doi.org/10.1136/bjo.2006.094250

Lueck, A. H., Dornbusch, H., \& Hart, J. (1999). The effects of training on a young child with cortical visual impairment: An exploratory study. Journal of Visual Impairment \& Blindness, 93, 778-793.

Lueck, A. H. (Ed). (2004). Functional vision: A practitioner's guide to evaluation \& intervention. New York, NY: AFB Press.

Lueck, A.H., Chen, D., Kekelis, L.S., \& Hartmann, E. (2008). Developmental guidelines for infants with visual impairments: A guidebook for early intervention. Second edition. Louisville, KY: American Printing House for the Blind.

Lueck, A.H., \& Dutton, G.N. (Eds.), (2015a). Vision and the brain: Understanding cerebral visual impairment in children. New York, NY: AFB Press.

Lueck, A.H., \& Dutton, G.N. (2015b). Assessment of children with CVI: Introduction and overview. In A.H. Lueck \& G.N. Dutton (Eds.), Vision and the brain: Understanding cerebral visual impairment in children (pp 207-260). New York, NY: AFB Press.

Lueck A.H., \& Dutton, G.N. (2015c). Intervention Methods: Overview and principles. In A.H. Lueck \& G.N. Dutton (Eds.), Vision and the brain: Understanding cerebral visual impairment in children. (pp 497-536). New York, NY: AFB Press.

Macintyre-Beon, C., Young, D., Calvert, J., Ibrahim, H., Dutton, G.N., \& Bowman, R. (2012). Reliability of a question inventory for structured history taking in children with cerebral visual impairment. Eye. 26, 1393. https://doi:10.1038/eye.2012.154

Mazeau, M. (2005). Neuropsychologie et troubles des apprentissages: Du symptôme à la réeducation. Paris: Elsevier Masson.

This is a provisional file, not the final typeset article 
McKyton, A., Ben-Zion, I., Doron, R., \& Zohary, E. (2015). The limits of shape recognition following late emergence from blindness. Current Biology, 25, 2373-2378. https://doi.org/10.1016/j.cub.2015.06.040

Mitry, D., Williams, C., Northstone, K., Akter A., Jewel, J., Khan, N., Muhit, M., Gilbert, C.E., \& Bowman, R. (2016). Perceptual visual dysfunction, physical impairment and quality of life in Bangladeshi children with cerebral palsy. British Journal of Ophthalmology. 100, 1245-1250. https://doi: 10.1136/bjophthalmol-2015-307296

Morash, V. S., \& McKerracher, A., (2017). Low reliability of sighted-normed verbal assessment scores when administered to children with visual impairments. Psychological Assessment, 29, 343348. http://dx.doi.org/10.1037/pas0000341

Morse, M.T., \& Morse, J.L. (2015). Supports for children and families. In A.H. Lueck \& G.N. Dutton (Eds.), Vision and the brain: Understanding cerebral visual impairment in children (pp 618645). New York, NY: AFB Press.

Nokleby, H. (2011). Triangulation with diverse intentions. Kapet. Karlstad University's Educational Journal, 7, 140-156. Retrieved from www.divaportal.org/smash/get/diva2:490569/FULLTEXT01.pdf. 7 October 2017.

Ortibus, E., Lagae, L., Casteels, I., Demaerel, P., \& Stiers, P. (2009). Assessment of cerebral visual impairment with the L94visual perceptual battery; Clinical value and correlation with MRI findings. Developmental Medicine \& Child Neurology, 51, 209-217. https://doi.org/10.1111/j.1469$\underline{8749.2008 .03175 . \mathrm{x}}$

Ortibus, E. L., DeCock, P. P., \& Lagae, L. G. (2011). Visual perception in preterm children: What are we currently measuring? Pediatric Neurology, 45, 1-10. https://doi.org/10.1016/j.pediatrneurol.2011.02.008

Ortibus, E., Pierens, G., Schoolmeesters, B., VanParijs, K., Janssens, E. (2016). G(anspoel). CVI.Tod(dler)s: A novel diagnostic tool in the assessment of cerebral visual impairment in the young child. Developmental Medicine and Child Neurology, 58 (S6) pp. 49. http://onlinelibrary.wiley.com/doi/10.1111/dmcn.13320/epdf

Pagliano, E., Fedrizzi, E., Erbetta, A., Bulgheroni, S., Solari, A., Bono, R., Fazzi, E., Andreucci, E., Riva, D. (2007). Cognitive profiles and visuoperceptual abilities in preterm and term spastic diplegic children with periventricular leukomalacia. Journal of Child Neurology. 22, 282-288. https://doi.org/10.1177/0883073807300529

Patton, M. Q. (2002). Qualitative Research \& Evaluation Methods. 3rd Ed. Thousand Oaks, CA: Sage Publications.

Pawletko, T., Chokon, S., \& Dutton, G.N., (2015). Considerations in the behavioral diagnosis of CVI: Issues, cautions, and potential outcomes. In A.H. Lueck \& G.N. Dutton (Eds.), Vision and the brain: Understanding cerebral visual impairment in children (pp. 145-17). New York, NY: AFB Press. 
Profiling children with cerebral visual impairment

885

886

887

888

889

890

891

892

893

894

895

896

897

898

899

900

901

902

903

904

905

906

907

908

909

910

911

912

913

914

915

916

917

918

919

920

921

922

923

924

925

926

927

928

929

930

931

932

933

Pel, J.J., Dudink, J., Vonk, M., Plaisier, A., Reiss, I.K., \& van der Steen J. (2016). Early identification of cerebral visual impairments in infants born extremely preterm. Developmental Medicine Child Neurology, 58, 1030-1035. https://doi.org/10.1111/dmcn.13115

Pennington, B.F. (2009). How neuropsychology informs our understanding of developmental disorders. Journal of Child Psychology \& Psychiatry, 50, 72-78. https://doi.org/10.1111/j.1469$\underline{7610.2008 .01977 . \mathrm{x}}$

Piaget, J. (1952). The origins of intelligence in children. New York, NY: International University Press. https://doi.org/10.1037/11494-000

Piaget, J. (1954). The construction of reality in the child. New York, NY: Basic Books. https://doi.org/10.1037/11168-000

Piaget, J. (1970). L’Epistémologie génétique, Paris, PUF.

Roman, C. (2010). CVI complexity sequences. Louisville, KY: American Printing House for the Blind.

Roman-Lantzy, C. (2007). Cortical Visual Impairment. New York: AFB Press.

Ruiter, S., Nakken, H., Janssen, M., Van Der Meulen, B., Looijestijn, P. (2011). Adaptive assessment of young children with visual impairment. British Journal of Visual Impairment, 29, 93112. https://doi.org/10.1177/0264619611402766

Sabatinelli, D., Lang, P.J., Bradley, M.M., Costa, V.D., Keil, A. (2009). The timing of emotional discrimination in human amygdala and ventral visual cortex. Journal of Neuroscience, 29, 1486414868. https://doi.org/10.1523/JNEUROSCI.3278-09.2009

Salvia, J.E., Ysseldyke, J., \& Bolt, S. (2013). Assessment in special and inclusive education (12th ed.). Belmont, CA: Wadsworth Publishing.

Shafer, S.Q., Stokman, C.J., Shaffer, D., Ng, S.K., O'Connor, P.A., Schonfeld, I.S. (1986). Tenyear consistency in neurological test performance of children without focal neurological deficit. Developmental Medicine \& Child Neurology, 28, 417-27.https://doi.org/ 10.1111/j.14698749.1986.tb14279.x

Sonksen, P.M. (1993). The assessment of vision in the preschool child. 68, 513-516. https://doi.org/10.1136/adc.68.4.513

Sonksen, P.M., \& Dale, N. (2002). Visual impairment in infancy: Impact on neurodevelopmental and neurobiological processes. Developmental Medicine \& Child Neurology, 44, 782-791. https://doi.org/10.1111/j.1469-8749.2002.tb00287.x

Steendam, M. (2015). Assessment linked to intervention: Observational assessment of young children and children with multiple disabilities. In A.H. Lueck \& G.N. Dutton (Eds.), Vision and the brain: Understanding cerebral visual impairment in children (pp. $391-410)$. New York, NY: AFB Press.

This is a provisional file, not the final typeset article 
Profiling children with cerebral visual impairment

934

935

936

937

938

939

940

941

942

943

944

945

946

947

948

949

950

951

952

953

954

955

956

957

958

959

960

961

962

963

964

965

966

967

968

969

970

971

972

973

974

975

976

977

978

979

980

981

982
Stiers, P., Vanderkelen, R., Vannester, G., Coene, S., Rammelaere, M., Vandenbussche, E. (2002). Visual-perceptual impairment in a random sample of children with cerebral Palsy. Developmental Medicine \& Child Neurology. 44, 370-382. https://doi.org10.1111/j.14698749.2002.tb00831.x

Struble, R.D. Jr., House, R., Trower, P.T., Lawrence, L.M. (2016). Efficacy of a vision- screening tool for birth to 3 years early intervention programs. Journal of the American Association of Pediatric Ophthalmology \& Strabismus, 20, 431-434. http://dx.doi.org/10.1016/j.jaapos.2016.06.005

Tietjen, M. (2016). "What's the complexity: A framework for addressing the complexity of visual demands throughout the school day for students with cortical visual impairment (CVI)." American Conference on Pediatric Cortical Visual Impairment, 9 July, 2016. Omaha Marriott, Omaha, NE. Conference Presentation.

Tröster H., \& Brambring M., (1992). Early social-emotional development in blind infants. Child: Care, Health and Development, 18, 207-227. https://doi.org/10.1111/j.1469-8749.2002.tb00287.x

Vancleef, K., Petre, Y., Janssens, E., Baumer, S., Ortibus, E., \& Wagemans, J. (2015). CVIT 3-6, a screening test for cerebral visual impairment in young children. Perception, Supplement 44, 77-78. Abstract retrieved from http://journals.sagepub.com/doi/pdf/10.1177/0301006615598674

van Genderen, M., Dekker, M., Pilon, F., \& Bals, I. (2012). Diagnosing cerebral visual impairment in children with good visual acuity. Strabismus, 20, 78-83.

https://doi.org/10.3109/09273972.2012.680232

Watson, T., Orel-Bixler, D., Haegerstrom-Portnoy, G. (2010). Early visual-evoked potential acuity and future behavioral acuity in cortical visual impairment. Optometry \& Visual Science, 87, 80-86. https://doi.org/10.1097/OPX.0b013e3181c75184

Walthes, R., Freitag, C., \& Jacob (2015). Cerebral visual impairment in children. Perception, Supplement 44, p. 77. Abstract retrieved from http://journals.sagepub.com/doi/abs/10.1177/0301006615598674

Warren, D.H. (1994). Blindness and children: A developmental differences approach. New York, NY: Cambridge University Press. https://doi.org/10.1017/CBO9780511582288

Ysseldyke, J., Salvia, J. E., \& Bolt, S., (2009). Assessment in special and inclusive education (11th ed.), Belmont, CA: Wadsworth Publishing.

Zhang, X., Kedar, S. Lynn, M.M., Newman, M.D., \& Blousse, M.D. (2006a) Homonymous hemianopia: Clinical-anatomic correlations in 904 cases. Neurology, 66, 906-910.

https://doi.org/10.1212/01.wnl.0000203913.12088.93

Zhang, X., Kedar, S., Lynn, M. J., Newman, N. J., \& Blousse, V. (2006b). Natural history of homonymous hemianopia. Neurology, 66, 901-905.

https://doi.org/10.1212/01.wnl.0000203338.54323.22

Zihl, J., \& Dutton, G.N. (2015). Cerebral visual impairment in children: Visuoperceptive and visuocognitive disorders. New York: Springer. https://doi.org/10.1007/978-3-7091-1815-3 
983

984

985

986

987

988

989

990

991

992

993

994
Zuidhoek, S., (2015). The role of attention and executive brain functions in seeing and behavior in children with CVI. In A.H. Lueck \& G.N. Dutton (Eds.), Vision and the brain: Understanding cerebral visual impairment in children (pp. 124-144). New York, NY: AFB Press.

Zuidhoek, S., Hyvarinen, L., Jacob, N., \& Henriksen, A. (2015). Assessment of functional vision: Visual processing of children with CVI. In A.H. Lueck \& G.N. Dutton (Eds.), Vision and the brain: Understanding cerebral visual impairment in children (pp. 343-390). New York, NY: AFB Press. 